\title{
L-Cyclic Magma versus R-Cyclic Magma
}

\author{
U.Ananda Reddy ${ }^{1}$, Dr.G.Shobhalatha ${ }^{2}$, Dr.L.Sreenivasulu Reddy ${ }^{3}$ \\ ${ }^{1}$ Reseach Scholar, Department of Mathematics, S K University, Ananthapurum,A.P,India \\ ${ }^{2}$ Professor, Department of Mathematics, S K University, Ananthapurum,A.P,India. \\ ${ }^{3}$ Academic Consultant, Department of Mathematics, S V University, Tiruapti,India.
}

\begin{abstract}
A mapping $*: X \times X \rightarrow X$ is a (binary) operation, and the pair $(X, *)$ is named as a Magma [1]. Magma with the property: $x *(y * z)=z *(x * y)=y *(z * x)$ for all $x, y, z$ in Magma is named as L-cyclic magma or with the property $(x * y) * z=(z * x) * y=(y * z) * x$ for all $x, y, z$ in Magma is named as $R$ cyclic magma. In this paper, every result show only identical gaol, that is, to prove when a L-cyclic magma becomes $R$-cyclic magma and vice versa.
\end{abstract}

Keywords: Magma, L-cyclic magma, R-cyclic magma, L-R-cyclic magma, L-identity, R-identity and cross cancellation law.

\section{INTRODUCTION}

The algebraic objects encountered in this chapter are sets with a binary operation defined on them. Andreas[1] introduced a term "magma" in his Ph.D., theses with entitle "Classification and Enumeration of finite semigroups". Magma nothing but an algebraic structure with one binary operation on a nonempty set. Throughout this paper, we consider the magma with atleast any one of the property

i. $\quad x *(y * z)=z *(x * y)=y *(z * x)$ for all $x, y, z$ in Magma

or

ii. $\quad(x * y) * z=(z * x) * y=(y * z) * x$ for all $x, y, z$ in Magma

The magma with first property is named as L-cyclic magma, with second property is named as Rcyclic magma. If it has both properties, then it is named as L-R-cyclic magma.

This paper contains two sections: In section 1, it contains the introduction and in section 2 shows the results when a L-cyclic magma becomes R-cyclic magma and vice versa.

\section{L-CYClic Magma Becomes R-CyClic Magma and Vice Versa}

In this section contains all necessary and sufficient conditions of L-cyclic magma becomes R-cyclic magma by using additional property: "commutative, left cancellation, right cancellation right identity, left identity or idempotent" on it.

Result 2.1: Let $(S, *)$ be a commutative magma. Then $(S, *)$ is L-cyclic magma if and only if it is $\mathrm{R}$ cyclic magma.

\section{Proof:}

Let $(S, *)$ be a L-cyclic magma $(S, *)$.

Consider, $(x * y) * z$

By using commutative property on $(x * y) * z$, we get $(x * y) * z=z *(x * y)$

By using cyclic property on $z *(x * y)$, we get $z *(x * y)=y *(z * x)$

By using commutative property on $y *(z * x)$, so $y *(z * x)=(z * x) * y$

Once again by using cyclic property on $*(z * x)$, so $y *(z * x)=x *(y * z)$ 
By using commutative property on $x *(y * z)$, so $x *(y * z)=(y * z) * x$

Thus $(x * y) * z=(z * x) * y=(y * z) * x$ for all $x, y, z$ in $S$.

Conversely, Consider $x *(y * z)$ in R-cyclic magma $(S, *)$.

Similarly by applying commutative and R-cyclic properties on $x *(y * z)$, we have $x *(y * z)=z *(x * y)=y *(z * x)$ for any $x, y, z$ in $S$.

Result 2.2: let $(S, *)$ be a magma with L-cancellation property. Then $(S, *)$ is L-cyclic magma if and only if it is R-cyclic magma.

\section{Proof:}

Since magma $(S, *)$ is L-cyclic magma, so $x *(x * y)=y *(x * x)=x *(y * x)$, for any $x, y$ in $S$.

That is $x *(x * y)=x *(y * x)$

Since magma $(S, *)$ has L-cancellation property, so $x *(x * y)=x *(y * x) \Rightarrow x * y=y * x$

Thus, $x * y=y * x$ for any $x, y$ in $S$.

Hence $(S, *)$ is a commutative magma.

By using result $2.1,(S, *)$ is R-cyclic magma.

Conversely,

Since magma $(S, *)$ is R-cyclic magma,

for any $x, y$ in $\mathrm{S},(x * x) *(x * y)=((x * y) * x) * x$.

$$
\begin{aligned}
& =((x * x) * y) * x \\
& =((y * x) * x) * x \\
& =(x *(y * x)) * x \\
& =(x * x) *(y * x)
\end{aligned}
$$

Since magma $(S, *)$ has L-cancellation property,

$$
\text { so }(x * x) *(x * y)=(x * x) *(y * x) \Rightarrow x * y=y * x
$$

Thus, $x * y=y * x$ for any $x, y$ in $S$.

Hence $(S, *)$ is a commutative magma.

By using result $2.1,(S, *)$ is R-cyclic magma.

Result 2.3: let $(S, *)$ be a magma with R-cancellation property. Then $(S, *)$ is L-cyclic magma if and only if it is R-cyclic magma.

\section{Proof:}

\section{Necessary Condition:}

Since magma $(S, *)$ is L-cyclic magma, for any $x, y$ in $\mathrm{S}$,

$$
\begin{aligned}
(x * y) *(x * x) & =x *((x * y) * x) \\
& =x *(x *(x * y)) \\
& =x *(y *(x * x)) \\
& =x *(x *(y * x)) \\
& =(y * x) *(x * x)
\end{aligned}
$$

Since magma $(S, *)$ has $\mathrm{R}$-cancellation property,

So $(x * y) *(x * x)=(y * x) *(x * x) \Rightarrow x * y=y * x$ 
Thus, $x * y=y * x$ for any $x, y$ in $S$.

Hence $(S, *)$ is a commutative magma.

By using result $2.1,(S, *)$ is R-cyclic magma.

\section{Sufficient condition:}

Since magma $(S, *)$ is R-cyclic magma,

for any $x, y$ in $\mathrm{S},(x * y) * x=(x * x) * y=(y * x) * x$

Since magma $(S, *)$ has R-cancellation property,

so $(x * y) * x=(y * x) * x \Rightarrow x * y=y * x$

Thus, $x * y=y * x$ for any $x, y$ in $S$.

Hence $(S, *)$ is a commutative magma.

By using result $2.1,(S, *)$ is L-cyclic magma.

Note: From above two results, it is understood that every L-cyclic magma is R-cyclic magma and vice versa if magma with cancellation property.

Result 2.4: let $(S, *)$ be a magma with R-identity. Then $(S, *)$ is L-cyclic magma if and only if it is $\mathrm{R}$ cyclic magma.

\section{Proof:}

Since $(S, *)$ magma has R-identity, so there exist an element $e$ in $S$, such that $x * e=x$ for all $x$ in $S$.

\section{Necessary Condition:}

Since the magma $(S, *)$ has L-cyclic magma,

so it has L-cyclic property $x *(y * z)=z *(x * y)=y *(z * x)$ for all $x, y, z$ in $S$.

Thus

$$
\begin{aligned}
x * y=(x * e) *(y * e) & \\
& =e *((x * e) * y) \\
& =y *(e *(x * e)) \\
& =y *(e *(e * x)) \\
= & y *(x *(e * e)) \\
= & y *(x * e) \\
= & y * x
\end{aligned}
$$

Thus the magma $(S, *)$ is commutative magma.

By using result $2.1,(S, *)$ is R-cyclic magma.

\section{Sufficient Condition:}

Since the magma $(S, *)$ has R-cyclic magma,

so it has $(x * y) * z=(z * x) * y=(y * z) * x$ for all $x, y, z$ in $S$.

Thus $\quad x * y=(x * e) *(y * e)$

$$
\begin{aligned}
& =((y * e) * x) * e \\
= & (y * x) * e \\
= & y * x
\end{aligned}
$$

Thus the magma $(S, *)$ is commutative magma.

By using result $2.1,(S, *)$ is L-cyclic magma. 
Result 2.5: let $(S, *)$ be a magma with L-identity. Then $(S, *)$ is L-cyclic magma if and only if it is R-cyclic magma.

\section{Proof:}

Since $(S, *)$ magma has L-identity, so there exist an element $e$ in $S$, such that $e * x=x$ for all $x$ in $S$.

\section{Necessary Condition:}

Since the magma $(S, *)$ has L-cyclic magma,

so it has L-cyclic property $x *(y * z)=z *(x * y)=y *(z * x)$ for all $x, y, z$ in $S$.

Thus $\quad x * y=(e * x) *(e * y)$

$$
\begin{aligned}
& =y *((e * x) * e) \\
& =y *((x * e)) \\
& =e *(y * x) \\
& =y * x
\end{aligned}
$$

Thus the magma $(S, *)$ is commutative magma.

By using result $2.1,(S, *)$ is R-cyclic magma.

\section{Sufficient Condition:}

Since the magma $(S, *)$ has R-cyclic magma,

so it has R-cyclic property $(x * y) * z=(z * x) * y=(y * z) * x$ for all $x, y, z$ in $S$.

Thus $x * y=(e * x) *(e * y)$

$$
\begin{aligned}
& =((e * y) * e) * x \\
= & (y * e) * x \\
& =(y * x) * e \\
= & (e * y) * x \\
= & y * x
\end{aligned}
$$

Thus the magma $(S, *)$ is commutative magma.

By using result 2.1, $(S, *)$ is L-cyclic magma.

Note : from above two results, it is easily show that L-cyclic magma is R-cyclic magma and vice versa if magma with identity.

Result 2.6: A cross cancelation magma $(S, *)$ with idempotent element $e$.Then $(S, *)$ is L-cyclic magma if and only if it is R-Cyclic magma.

\section{Proof:}

Since the magma $(S, *)$ has a cross cancelation property, so $x * y=y * z \Rightarrow x=z$ for any $x, y, z$ in $S$

\section{Necessary Condition:}

Let $e$ be an idempotent element of magma $(S, *)$.

So, $e * e=e$

\section{Case 1:}

since $S$ has a cyclic property, so $x *(e * e)=x * e$ for all $x$ in $S$.

$\Rightarrow e *(x * e)=x * e$

$\Rightarrow$ since $S$ has a cross cancelation property, s o $x * e=x$, for all $x$ in $S$.

Thus idempotent element $e$ is a right identity in magma $(S, *)$. 


\section{Case 2:}

Consider and element $e * x$

By using left identity of case 1, this is equal to $e *(e * x)$

By using L-cyclic property on $e *(e * x)$, we have $e *(e * x)=x *(e * e)$

Using idempotent property of $e$ on $x *(e * e), x *(e * e)=x * e$

Thus, $e *(e * x)=x * e$

$\Rightarrow$ since $S$ has a cross cancelation property, s o $e * x=x$, for all $x$ in $S$.

Thus idempotent element $e$ is a left identity in magma $(S, *)$.

Hence, the idempotent element $e$ is identity in $(S, *)$.

Next to show that commutative property of $(S, *)$.

Let $x, y$ are any two elements in magma $(S, *)$.

$$
\begin{aligned}
x * y & =(e * x) *(e * y) \\
& =y *((e * x) * e) \\
& =e *(y *(e * x)) \\
& =e *(y * x) \quad(\text { since } \mathrm{e} \text { is an left identity of } \mathrm{S}) \\
& =y * x \quad(\text { since } \mathrm{e} \text { is an left identity of } \mathrm{S})
\end{aligned}
$$

Thus the magma $(S, *)$ is commutative magma

By using result $2.1,(S, *)$ is R-cyclic magma.

\section{Sufficient Condition:}

Next to show that it is L-cyclic magma.

Consider $x *(y * z)$

By using commutative property on $x *(y * z)$, so we have $x *(y * z)=(y * z) * x \quad$ Let $e$ be an idempotent element of magma $(S, *)$.

So, $e * e=e$

\section{Case 1:}

since $S$ has a cyclic property, so $(e * e) * x=e * x$ for all $x$ in $S$.

$\Rightarrow(x * e) * e=e * x$

$\Rightarrow(e * x) * e=e * x$, for all $x$ in.

$\Rightarrow$ since $S$ has a cross cancelation property, so $(e * x)=x$, for all $x$ in $S$.

Thus idempotent element $e$ is a left identity in magma $(S, *)$.

\section{Case 2:}

Consider and element $x * e$

By using left identity of case 1 , this is equal to $(e * x) * e$.

By using R-cyclic property on $(e * x) * e$, we have $(e * x) * e=(e * e) * x$

Again twice by using left identity of case 1, we have $(e * e) * x=e * x \& e * x=x$

Thus, $x * e=x$

Next to show that $(S, *)$ is commutative magma.

\section{Commutative property:}

Let $x, y$ are any two elements in magma $(S, *)$. 


$$
\begin{aligned}
x * y & =(e * x) *(e * y) \\
& =((e * y) * e) * x \\
& =((e * e) * y) * x \\
& =(e * y) * x \\
& =y * x
\end{aligned}
$$

Thus the magma $(S, *)$ is commutative magma.

By using result $2.1,(S, *)$ is L-cyclic magma.

\section{REFERENCES}

[1] Andreas Distler of his theses "Classification and Enumeration of Finite Semigroups" submitted in the University of St Andrews, May 2010.

[2] Clifford, A.H., Preston, G.B.: The Algebraic Theory of Semigroups, I. Am. Math, Soc., Providence (1961).

[3] G.I.Moghaddam, R.Padmanabhan, "Cancellative Semigroups Admitting Conjugates" J.Semigroup Theory Appl.2015,2015:9, ISSN:2015-2937.

[4] Howie, J.M.: An Introduction to Semigroup Theory. Academic Press, San Diego (1976).

[5] Lehmer, D.H.: A ternary analogue of Abelian groups. Am. J. Math. 54, 329-338 (1932).

[6] Mayberg, K.: Lecture on Algebras and Triple Systems. Lecture Notes. Univ. of Virginia, Charlottesville (1972).

[7] M.L. Santiago· S. Sri Bala, “Ternary semigroups”, Semigroup Forum (2010) 81:380-388.

\section{AUTHOR'S BIOGRAPHY}

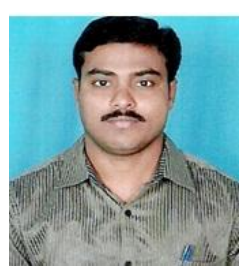

U.Ananda Reddy, presently working as a Assistant professor in Mathematics in Sir vishveshwaraiah institute of science and technology, Madanapalli,Andhra Pradesh, India. He is doing part time Ph.D in Mathematics in Sri Krishnadevaraya University, Anantapur,Andhra Pradesh, India under the guidance of Dr..G.Shobhalatha, Professor in Mathematics, in Sri Krishnadevaraya University. He is presented and participated many National and International conferences and workshops.

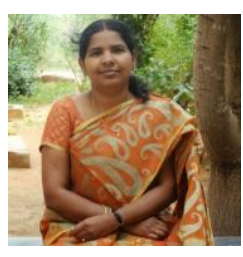

Prof. G. Shobhalatha, presently working as a Professor of Mathematics, Anantapur, Andhra Pradesh P, India. She has 13 years of Teaching perience. She Guided 11Ph.D's., and 10 M. Phil's., awarded. Presently four students for Ph.D and two students for M.Phil are working. She published 45 papers in national and international journals and participated many national and international seminars, conferences, symposia and Workshops.

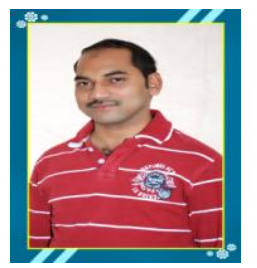

Dr.L.Sreenivasulu Reddy, presently working as Teaching Assistant in the Department of Mathematics at Sri Venkateswara University, Tirupati, Andra Pradesh, India. He was born on 10th Jun 1983 at Kadapa in Andra Pradesh. He completed his Graduation and Post graduation in Mathematics at Sri Venkateswara University, Tirupati. He got Ph.D from Sri Venkateswara University in the discipline of Mathematics in the year of 2009. He is well versed scholar in cryptography which of the thirst areas of Mathematics. He has been paying attention on Cryptography, Coding theory, Boolean algebra, Semigroup theory, Ring theory and Graph theory. His specializations are Cryptography, Semigroup theory, Ring theory and Graph theory. He has been trying to apply interrelationships between Cryptography and Algebraic Number theory, Semi group theory and Boolean algebra, Cayley Graphs and Arithmetic Graphs. He participated and presented papers in the area of cryptography, Graph theory and Semigroup theory in various international and national conferences. 